\title{
Fluorine-18-labeled boronophenylalanine positron emission tomography for oral cancers: Qualitative and quantitative analyses of malignant tumors and normal structures in oral and maxillofacial regions
}

\author{
YASUNORI ARIYOSHI ${ }^{1}$, MASASHI SHIMAHARA ${ }^{1}$, YOSHIHIRO KIMURA $^{1}$, YUICHI ITO ${ }^{1}$, \\ TAKESHI SHIMAHARA ${ }^{1}$, SHIN-ICHI MIYATAKE ${ }^{2}$ and SHINJI KAWABATA ${ }^{2}$ \\ ${ }^{1}$ Department of Dentistry and Oral Surgery, Division of Medicine for Function and Morphology of Sensory \\ Organs; ${ }^{2}$ Department of Neurosurgery, Division of Surgery, Osaka Medical College, Osaka, Japan
}

Received November 11, 2010; Accepted February 24, 2011

DOI: $10.3892 / \mathrm{ol} .2011 .265$

\begin{abstract}
The present study aimed to demonstrate the features of fluorine-18-labeled boronophenylalanine positron emission tomography ( $\left.{ }^{18} \mathrm{~F}-\mathrm{BPA}-\mathrm{PET}\right)$ to reveal oral cancer, as well as normal structures in the oral and maxillofacial regions. We analyzed ${ }^{18} \mathrm{~F}$-BPA-PET findings from 8 patients with histologically confirmed recurrent and/or advanced oral cancer scheduled for boron neutron capture therapy. The capacity of ${ }^{18} \mathrm{~F}$-BPA-PET to delineate tumor and normal structures was assessed qualitatively and quantitatively. Tumors were easily identified as high uptake areas in all cases. Although the eyes, which were depicted as a low uptake area, and tongue musculature were readily identified, major vessels were not noted in any of the cases. Areas corresponding to the surface of the dorsum tongue to middle pharynx were expressed as high uptake areas in all of the cases. Quantitatively, tumors were expressed as the highest uptake area in 6 of the 8 cases, while the dorsum tongue had the highest uptake area in the remaining 2 cases. ${ }^{18} \mathrm{~F}$-BPA-PET is useful in demonstrating the presence of a tumor. Thus, it is crucial to note the presence of a high uptake area corresponding to the dorsum area of the tongue when diagnosing a tumor using this technique.
\end{abstract}

\section{Introduction}

Fluorodeoxyglucose (FDG) positron emission tomography (PET) has recently become widely used for detecting malignant tumors of the oral cavity, particularly for the diagnosis

Correspondence to: Dr Yasunori Ariyoshi, Department of Dentistry and Oral Surgery, Division of Medicine for Function and Morphology of Sensory Organs, 2-7 Daigaku-machi, Takatsuki, Osaka 569-8686, Japan

E-mail: ora009@poh.osaka-med.ac.jp

Key words: fluorine-18-labeled boronophenylalanine positron emission tomography, oral cancer, boron neutron capture therapy of metastatic deposits (1). In addition, another type of PET method, methionine-basis PET, has been shown to be useful for diagnosing malignancies (2).

Boron neutron capture therapy (BNCT) was recently performed in order to regulate non-resectable advanced and/or recurrent oral cancers, and its clinical utility was previously reported $(3,4)$. However, certain patients suffer from alopecia and/or mucositis after undergoing BNCT $(3,5)$. For planning, as well as predicting the outcome and adverse effects of this therapy, boron concentrations should be evaluated in surrounding normal structures as well as in the tumor itself, for which fluorine-18-labeled boronophenylalanine ( $\left.{ }^{18} \mathrm{~F}-\mathrm{BPA}\right)$ PET was utilized. To the best of our knowledge, features of normal tissues and tumors in ${ }^{18} \mathrm{~F}$-BPA-PET images have yet to be adequately assessed. The present study therefore aimed to qualitatively and quantitatively elucidate the features of normal structures in the oral and maxillofacial regions, as well as associated tumors revealed by ${ }^{18} \mathrm{~F}-\mathrm{BPA}-\mathrm{PET}$ imaging.

\section{Materials and methods}

The study evaluated ${ }^{18} \mathrm{~F}$-BPA-PET images obtained in examinations of 8 patients with histologically confirmed recurrent and/or advanced oral cancer, or metastatic lymphadenopathy who were scheduled for BNCT (Table I). Seven of the patients were treated with a combination of surgery, chemotherapy and radiotherapy, while 1 patient was treated with thermotherapy. All PET examinations were performed using an ECAT ACCEL (Siemens Japan Co., Tokyo, Japan) with ${ }^{18}$ F-labeled BPA (37-55.5 MBq/10 kg of body weight) injected 45 min prior to obtaining the images, which were analyzed with AMIDE software (SourceForge, Inc., Mountain View, CA, USA). Signed informed consent was obtained from each patient prior to the PET examinations.

All images were assessed by detecting high uptake areas in the head and face, including the oral cavity by experts who did not have access to the personal and clinical data of the patients, including age, gender, affected site, tumor stage and previous treatment. Following assessment of the high uptake 
Table I. Patient characteristics.

\begin{tabular}{|c|c|c|c|c|c|}
\hline Case & $\begin{array}{c}\text { Age } \\
\text { (years) }\end{array}$ & Gender & Location & Histopathological diagnosis & Previous treatment \\
\hline 1 & 57 & Male & Maxilla & Squamous cell carcinoma & Surgery, chemotherapy, radiotherapy \\
\hline 2 & 67 & Female & Maxilla & Mucoepidermoid carcinoma & Surgery, chemotherapy, radiotherapy \\
\hline 3 & 70 & Female & Maxilla & Adenocarcinoma & Surgery, chemotherapy, radiotherapy \\
\hline 4 & 74 & Female & Upper lip & Adenocarcinoma & Thermotherapy \\
\hline 5 & 82 & Male & Oral floor & Adenoid cystic carcinoma & Surgery, chemotherapy, radiotherapy \\
\hline 6 & 62 & Male & Oral floor & Squamous cell carcinoma & Surgery, chemotherapy, radiotherapy \\
\hline 7 & 74 & Female & Cervical lymph node & Squamous cell carcinoma & Surgery, chemotherapy, radiotherapy \\
\hline 8 & 32 & Male & Cervical lymph node & Malignant melanoma & Surgery, chemotherapy \\
\hline
\end{tabular}

Table II. Qualitative analysis of the high uptake areas.

\begin{tabular}{lll}
\hline Case & \multicolumn{1}{c}{ Tumor site } & \multicolumn{1}{c}{ High uptake area } \\
\hline 1 & Left maxilla & Left maxilla, right posterior portion of dorsum tongue \\
2 & Right maxilla & Right maxilla, medial portion of dorsum tongue \\
3 & Anterior maxilla & Anterior maxilla, medial posterior portion of dorsum tongue \\
4 & Medial upper lip & Medial upper lip area, medial anterior portion of dorsum tongue \\
5 & Right sublingual gland & Right sublingual area, left posterior portion of dorsum tongue \\
6 & Anterior oral floor & Medial anterior oral floor, medial and lateral posterior portion of the dorsum tongue \\
7 & Submental region & Submental area, medial anterior portion of the tongue \\
8 & Right jugulodigastric node & Right upper neck area, medial posterior portion of the dorsum tongue
\end{tabular}

areas, the evaluators were provided information regarding only the site of the tumor. The investigators assessed the images for identification of the brain, ventricle, eyes, parotid and submandibular glands, major vessels, maxillary sinus and tongue musculature with regards to the ability of ${ }^{18} \mathrm{~F}-\mathrm{BPA}-\mathrm{PET}$ for identification. Each of these objects was then classified as identified or not identified. In addition, other specific features noted were freely documented. All bilateral structures on the contralateral site of the tumor were also assessed, since some of the patients underwent surgery, such as neck dissection, maxillectomy, or other radical surgical treatment in the affected site, and some anatomical structures were not present. For these analyses, all axial, coronal and sagittal images were freely investigated independently by three oral surgeons. In addition, computed tomography (CT) and magnetic resonance image (MRI) examinations were used to detect tumor localization. For qualitative analyses the structures were classified as identified only when all 3 evaluators identified each structure.

Following the qualitative investigations, the region of interest (ROI) was placed in the tumor as well as in the normal structures. The choice of field of view (FOV) was independently assessed by the 3 oral surgeons, and the results were averaged. The ratios of uptake of boron in the tumor as well as in each normal structure to that of the brain were calculated according to the formulas:

Tumor to brain $(\mathrm{T} / \mathrm{B})$ ratio = radioactivity of the tumor/ radioactivity of the brain.

Normal tissue to brain $(\mathrm{N} / \mathrm{B})$ ratio = radioactivity of normal tissue/radioactivity of the brain.

\section{Results}

Qualitative evaluation of high uptake areas. In all patients, high uptake areas were precisely correlated to the actual tumor sites (Fig. 1), while another high uptake area was found in the oral cavity, particularly around the tongue, in all cases (Fig. 2). In 5 of the patients, this high uptake portion in the oral cavity was the area corresponding to the medial portion of the dorsum tongue, while that of 2 cases was the lateral area of the dorsum tongue. In 7 patients with a tumor in the oral cavity, 2 areas of high uptake were independently expressed in 1 case (Case 1) and continuously expressed in 6 (Fig. 3). These high uptake areas located around the tongue were easily detected in both sagittal and coronal scans, as compared to axial scans (Table II). The skin of the head, particularly the parietal, occipital, and temporal regions, including the entire field of the calvarium, was found to be an intermediate uptake area in all cases (Fig. 1).

Detection of anatomical structures. The area corresponding to the brain was expressed as a low to intermediate uptake area in all cases (Fig. 3A). The ventricle, identified as a low uptake area, was differentiated from the brain in 7 cases (Fig. 1), while the eyes were regarded as low uptake areas in all cases. The parotid gland was identified as a symmetrical intermediate uptake area in 7 cases (Fig. 3B), whereas the submandibular glands were observed in only 2 cases. The maxillary sinus, jugular vein and carotid artery were not observed in any of the cases. The area corresponding to the oral cavity was regarded 


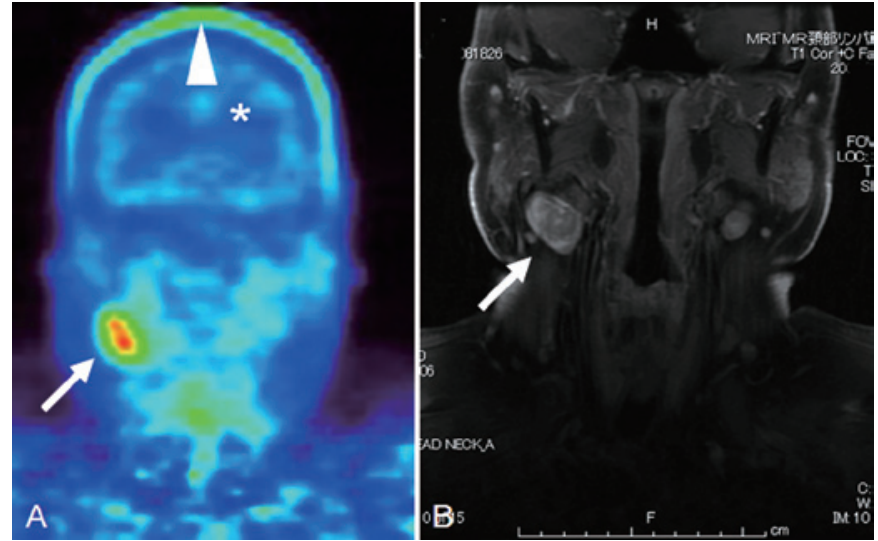

Figure 1. Metastatic malignant melanoma extending to the cervical lymph node (original site, right maxilla). (A) ${ }^{18} \mathrm{~F}-\mathrm{BPA}-\mathrm{PET}$ scan. A metastatic lymph node was indicated as a high uptake area (arrow) in ${ }^{18} \mathrm{~F}-\mathrm{BPA}-\mathrm{PET}$ scanning. The skin surface of the head showed an intermediate to high uptake of ${ }^{18} \mathrm{~F}-\mathrm{BPA}$ (arrowhead). The ventricle was differentiated from the brain parenchyma, which was depicted as a low uptake area $\left(^{*}\right)$. (B) Magnetic resonance image (gadolinium-enhanced fat-suppressed T1-weighted).

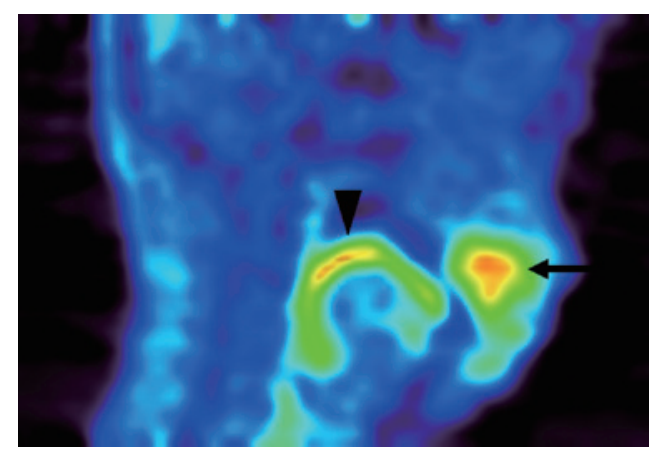

Figure 2. Adenocarcinoma in the anterior maxilla (Case 3). A tumor was located in the anterior maxilla and was expressed as a high uptake area in ${ }^{18}$ F-BPA-PET scanning (arrow). Another high uptake area (arrowhead) was noted in the posterior oral cavity, which correlated to the surface of the oral tongue and tongue base.

as a high uptake area as compared to other head and neck areas. Notably, an apparent high uptake area from the dorsum tongue to middle pharynx was evident in the sagittal and coronal scans (Fig. 2). In 5 cases, the area surrounding the jaw bone was shown as a high uptake area (Fig. 4, Table III).

Quantitative analysis of each structure. The T/B ratio was different in each case, ranging from 2.34 to 5.40 with an average of 3.25. According to our qualitative analysis, the brain, ventricle, eyes, parotid and submandibular glands, tongue musculature, and the high uptake area located around the tongue (dorsum tongue) were considered quantitative analysis. The average N/B ratio values for the ventricle $(n=7)$, eye $(n=8)$, parotid gland $(\mathrm{n}=7)$, submandibular gland $(\mathrm{n}=2)$, tongue $(\mathrm{n}=8)$ and dorsum tongue $(\mathrm{n}=8)$ were $0.49,0.39,1.56,1.75,1.45$, and 2.59 , respectively (Table IV).

\section{Discussion}

Clinical trials of BNCT have been performed mainly for highgrade gliomas (5) and cutaneous melanomas (6). However, the
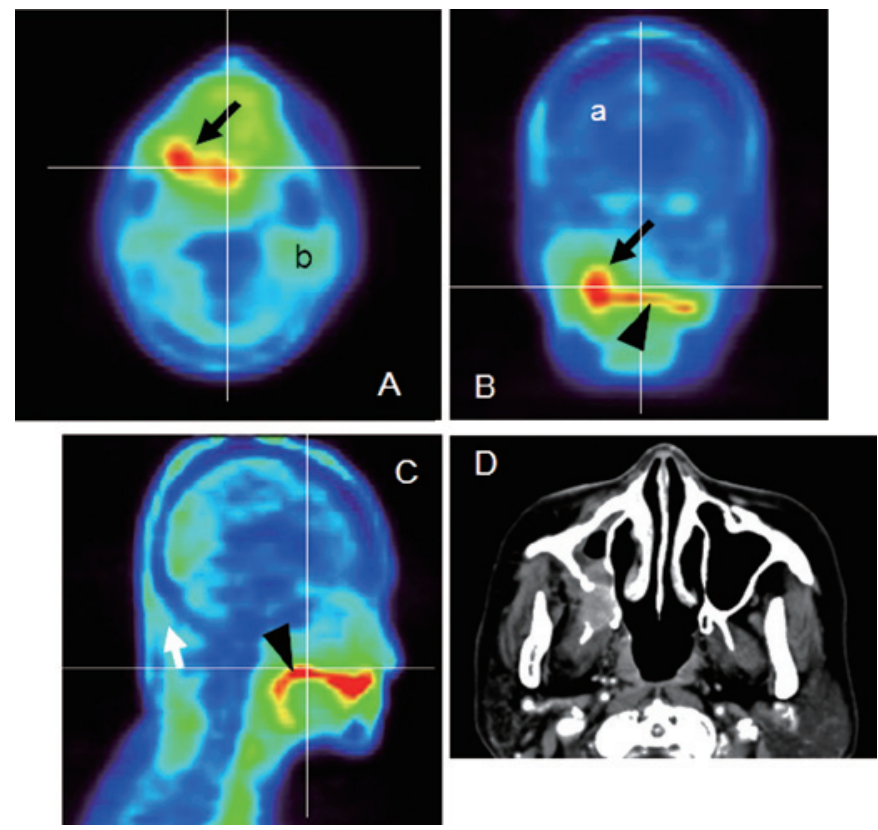

Figure 3. Mucoepidermoid carcinoma of the right maxilla (Case 2) (A) Axial, (B) coronal and (C) sagittal ${ }^{18} \mathrm{~F}$-BPA-PET. The horizontal and vertical lines correlate to the cut surface of each image. As normal structures, the brain parenchyma (a) and parotid gland (b) were easily detected. The tumor was expressed as a high uptake area (arrows), which continued to the high uptake area that was the dorsum surface of the tongue (arrowheads). Differentiation between the two structures was difficult on the ${ }^{18} \mathrm{~F}$-BPA-PET images. (D) Axial-enhanced CT.
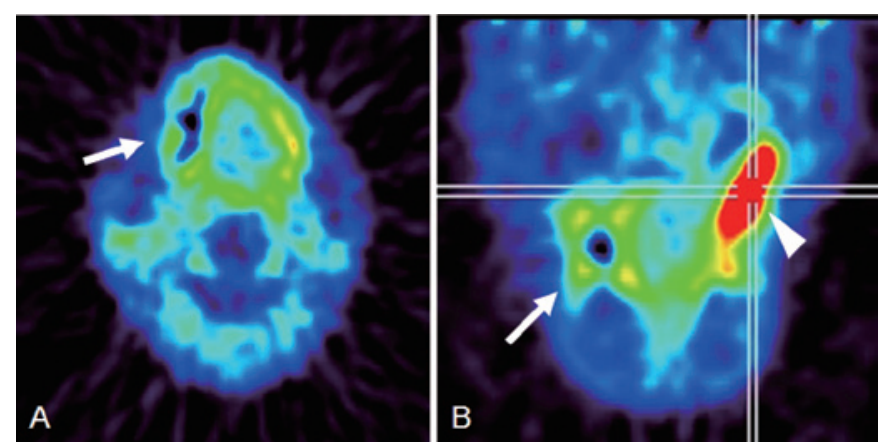

Figure 4. Squamous cell carcinoma of the left maxilla (Case 1). (A) Axial and (B) sagittal ${ }^{18} \mathrm{~F}$-BPA-PET. The area surrounding the jaw bone was identified as a high uptake area (arrows). The tumor was easily detected as an area of high uptake (arrowheads).

indication for BNCT has recently been broadened to include head and neck tumors (7), for which we previously reported its clinical utility (3). In theory, BNCT provides a means to selectively eradicate malignant cells and spare normal cells, although to ensure success, a sufficient amount of ${ }^{10} \mathrm{~B}$ should be selectively delivered to the tumor and an adequate number of thermal neutrons should be absorbed in order to sustain a lethal ${ }^{10} \mathrm{~B}(\mathrm{n}, \alpha){ }^{7} \mathrm{Li}$ capture reaction (8). Clinically, sodium borocaptate BSH and BPA have been used as boron delivery agents. Ono et al (9) reported that BPA accumulation occurs in cycling tumor cells but not in quiescent cells of solid tumors, while the therapeutic combination with BSH was a viable option for the issue of the heterogeneous microdistribution of BPA. In another study, Ono et al (10) reported that 
Table III. Detection of anatomical structures.

\begin{tabular}{lcccccccc}
\hline Case & Brain & Ventricle & Eyeball & $\begin{array}{c}\text { Parotid } \\
\text { gland }\end{array}$ & $\begin{array}{c}\text { Submandibular } \\
\text { gland }\end{array}$ & $\begin{array}{c}\text { Major } \\
\text { vessels }\end{array}$ & $\begin{array}{c}\text { Maxillary } \\
\text { sinus }\end{array}$ & $\begin{array}{c}\text { Tongue } \\
\text { musculature }\end{array}$ \\
\hline 1 & I & N & I & I & I & N & N & I \\
2 & I & I & I & I & N & N & N & I \\
3 & I & I & I & I & N & N & N & I \\
4 & I & I & I & I & N & N & N & I \\
5 & I & I & I & I & I & N & N & I \\
6 & I & I & I & I & N & N & N & I \\
7 & I & I & I & I & N & N & N & I \\
8 & I & I & I & N & N & N & N & I \\
\hline
\end{tabular}

I, identified; N, not identified.

Table IV. Quantitative evaluation of the tumor and anatomical structures.

\begin{tabular}{|c|c|c|c|c|c|c|c|}
\hline \multirow[b]{2}{*}{ Case } & \multirow[t]{2}{*}{$\mathrm{T} / \mathrm{B}$} & \multicolumn{6}{|c|}{ N/B } \\
\hline & & Ventricle & Eyeball & Parotid gland & Submandibular gland & Tongue & Dorsum tongue \\
\hline 1 & 5.40 & - & 0.17 & 1.78 & 2.09 & 1.45 & 2.57 \\
\hline 2 & 3.18 & 0.59 & 0.63 & 1.65 & - & 1.77 & 3.34 \\
\hline 3 & 3.91 & 0.39 & 0.28 & 1.71 & - & 1.53 & 3.88 \\
\hline 4 & 2.40 & 0.47 & 0.61 & 1.85 & - & 1.35 & 2.45 \\
\hline 5 & 3.08 & 0.27 & 0.14 & 1.70 & 1.41 & 1.57 & 2.31 \\
\hline 6 & 2.34 & 0.51 & 0.31 & 1.23 & - & 1.21 & 1.94 \\
\hline 7 & 2.84 & 0.45 & 0.54 & 0.97 & - & 1.62 & 2.13 \\
\hline 8 & 2.87 & 0.73 & 0.41 & - & - & 1.14 & 2.07 \\
\hline Average & 3.25 & 0.49 & 0.39 & 1.56 & 1.75 & 1.45 & 2.59 \\
\hline
\end{tabular}

$\mathrm{T} / \mathrm{B}$, tumor to brain ratio; N/B, normal tissue to brain ratio.

analternative solution was neutronirradiation during continuous injection of BPA, which we have employed clinically (3).

For BPA-based BNCT, it is necessary to analyze the actual distribution of BPA in vivo before determining factors for its indication, as well as before planning treatment and predicting outcome. For these purposes, numerous authors have used ${ }^{18} \mathrm{~F}-\mathrm{BPA}-\mathrm{PET}$ prior to employing BPA-based BNCT, and reported its usefulness (11-14). However, the possibility of detecting the spatial localization of the tumor as well as delineation of normal anatomical structures has yet to be confirmed. In the present study, some anatomical structures in the oral and maxillofacial region were routinely identified; the tongue and parotid gland were easily detected, with the exception of the parotid gland in case 8 . On the other hand, it was difficult to identify musculature, major vessels including the carotid artery and jugular vein, and maxillary sinuses, which were readily delineated in the same subjects with the use of CT and MRI. The utility of FDG-PET/CT for diagnosing head and neck cancers has been reported, and it was found that the method shows both anatomical and functional images simultaneously $(15,16)$. Additional investigations of PET/CT with fluoride- labeled BPA as a tracer are warranted, not only for improving the diagnostic potential, but also for treatment planning.

Kabalka et al (13) noted the importance of a significant uptake of ${ }^{18} \mathrm{~F}-\mathrm{BPA}$ in the oral mucosa and parotid glands, suggesting that patients who receive BNCT are likely to develop oral mucositis and/or parotitis if the epithermal neutron beam is near those structures. Busse et al (17) reported that BPA becomes concentrated, not only in the tumor, but also in rapidly growing normal tissues such as skin and mucosa of the oral cavity. Coderre et al (18) used Fisher 344 rats in an investigation and found that the oral mucosa was highly sensitive to BPA-mediated BNCT, indicating a dose-limiting effect for normal tissue when BNCT is applied for brain, and head and neck tumors. Notably, we found oral mucositis in patients who received BNCT for tumors in the oral cavity (3). In the present study, high uptake areas of ${ }^{18} \mathrm{~F}$-BPA were qualitatively detected in the tumor itself, as well as the medial portion of the oral cavity corresponding to the dorsum surface of the tongue and pharyngeal area, which were easily detected on sagittal and coronal scans. In addition, similar areas of high uptake were noted around the jaw bones 
(Fig. 4, arrows). In contrast, major salivary glands, including the parotid and submandibular glands, were found to be intermediate uptake areas. These findings suggest that high uptake areas in the oral cavity reflect, not only the oral mucosa, but also saliva. Of note is that this high uptake area in the oral cavity was detected in all cases, although the shapes varied. In addition, we found that quantitative and qualitative differentiation between these high signal areas and tumors based on the features of ${ }^{18} \mathrm{~F}$-BPA-PET was difficult as there were no apparent differences between the N/B and T/B ratios. In other words, the high uptake area corresponding to the dorsum tongue showed a higher N/B ratio as compared to that of the $\mathrm{T} / \mathrm{B}$ ratio in 2 of our 8 patients. It is crucial to be aware of the presence of this high uptake area when diagnosing a lesion located in the oral cavity using ${ }^{18} \mathrm{~F}$-BPA-PET and care must be taken to avoid misdiagnosis of a tumor.

Clinically, the adverse effects of BNCT are not as severe when compared to those of conventional radiotherapy $(3,7,19)$. Miyatake et al (5) reported that all of their patients who suffered from brain tumors exhibited alopecia, whereas no acute brain swelling or disturbance of consciousness occurred following BNCT. Moreover, Kato et al (7) reported few side effects, such as transient mucositis and alopecia, following BNCT therapy for head and neck tumors. We also noted alopecia and mucositis after BNCT (3). Alopecia is not a lifethreatening adverse effect, although it considerably decreases patient quality of life. Regarding the present findings, of note is that the skin of the head showed an intermediate to high uptake of ${ }^{18} \mathrm{~F}$-BPA in all patients whose head was included in the image (Fig. 1, arrowhead). This suggests that the skin was damaged by BNCT when included in the irradiation field. A novel technique to avoid accumulation of BPA in the skin of the head is required to decrease alopecia in patients who receive this type of therapy for head and neck tumors.

In their study, Miyashita et al (20) reported that differentiation between glioblastoma tumor progression and radiation necrosis can be achieved with ${ }^{18} \mathrm{~F}$-BPA-PET. These authors suggested repeat PET imaging to evaluate changes in tumor activity with respect to the treatment received. As for the patients included in the present study, a ${ }^{18} \mathrm{~F}$-BPA-PET study was not performed following BNCT. Assessment of followup ${ }^{18} \mathrm{~F}-\mathrm{BPA}-\mathrm{PET}$ for head and neck malignancies warrants further study.

\section{References}

1. Krabbe CA, Pruim J, Scholtens AM, Roodenburg JL, Brouwers AH, Phan TT, Agool A and Dijkstra PU: ${ }^{18}$ F-FDG-PET in squamous cell carcinoma of the oral cavity and oropharynx: a study on inter- and intraobserver agreement. J Oral Maxillofac Surg 68: 21-27, 2010.

2. Hsieh HJ, Lin SH, Lin KH, Lee CY, Chang CP and Wang SJ: The feasibility of ${ }^{11} \mathrm{C}$-methionine-PET in diagnosis of solitary lung nodule/masses when compared with ${ }^{18} \mathrm{~F}-\mathrm{FDG}-\mathrm{PET}$. Ann Nucl Med 22: 533-538, 2008.

3. Ariyoshi Y, Miyatake S, Kimura Y, Shimahara T, Kawabata S, Nagata K, Suzuki M, Maruhashi A, Ono K and Shimahara M: Boron neutron capture therapy using epithermal neutrons for recurrent cancer in the oral cavity and cervical lymph node metastasis. Oncol Rep 18: 861-866, 2007.

4. Kimura Y, Ariyoshi Y, Miyatake S, Shimahara M, Kawabata S and Ono K: Boron neutron capture therapy for papillary cystadenocarcinoma in the upper lip: A case report. Int J Oral Maxillofac Surg 38: 293-295, 2009.
5. Miyatake S, Kawabata S, Kajimoto Y, Aoki A, Yokoyama K, Yamada M, Kuroiwa T, Tsuji M, Imahori Y, Kirihata M, Sakurai Y, Masunaga S, Nagata K, Maruhashi A and Ono K: Modified boron neutron capture therapy for malignant gliomas performed using epithermal neutron and two boron compounds with different accumulation mechanisms: an efficacy study based on findings on neuroimages. J Neurosurg 103: 1000-1009, 2005.

6. Mishima Y, Honda C, Ichibashi M, Obara H, Hiratsuka J, Fukuda H, Karashima H, Kobayashi T, Kanda K and Yoshino K: Treatment of malignant melanoma by single neutron capture therapy with melanoma-seeking ${ }^{10} \mathrm{~B}$-compound. Lancet 2 : 388-389, 1989.

7. Kato I, Ono K, Sakurai Y, Ohmae M, Maruhashi A, Imahori Y, Kirihata M, Nakazawa M and Yura Y: Effectiveness of BNCT for recurrent head and neck malignancies. Appl Radiat Isotopes 61: 1069-1073, 2004.

8. Barth RF, Coderre JA, Vicente MG and Blue TE: Boron neutron capture therapy of cancer: Current status and future prospects. Clin Cancer Res 11: 3987-4002, 2005

9. Ono K, Masunaga S, Kinashi Y, Takagaki M, Akaboshi M, Kobayashi T and Akuta K: Radiological evidence suggesting heterogenous microdistribution of boron compounds in tumors: its relation to quiescent cell population and tumor cure in neutron capture therapy. Int J Radiat Oncol Biol Phys 34: 1081-1086, 1996.

10. Ono K, Masunaga S, Kinashi Y, Nagata K, Suzuki M, Sakurai Y, Maruhashi A, Kato I, Nakazawa M, Ariyoshi Y and Kimura Y: Neutron irradiation under continuous BPA injection for solving the problem of heterogenous distribution of BPA. In: Advances in Neutron Capture Therapy. Takamatsu, Plenum Press, New York, pp27-30, 2006.

11. Imahori Y, Ueda S, Ohmori Y, Sakae K, Kusuki T, Kobayashi T, Takagaki M, Ono K, Ido T and Fujii R: Positron emission tomography-based boron neutron capture therapy using boronophenylalanine for high-grade gliomas: Part II. Clin Cancer Res 4: 1833-1841, 1998.

12. Nichols TL, Kabalka GW, Miller LF, Khan MK and Smith GT: Improved treatment planning for boron neutron capture therapy for glioblastoma multiforme using fluorine-18 labeled boronophenylalanine and positron emission tomography. Med Phys 29: 2351-2358, 2002.

13. Kabalka GW, Nichols TL, Smith GT, Miller LF, Khan MK and Busse PM: The use of positron emission tomography to develop boron neutron capture therapy treatment plans for metastatic malignant melanoma. J Neurooncol 62: 187-195, 2003.

14. Havu-Auren K, Kiiski J, Lehtio K, Eskola O, Kulvik M, Vuorinen V, Oikonen V, Vahatalo J, Jaaskelainen J and Minn H: Uptake of 4-borono-2-(18F)fluoro-L-phenylalanine in sporadic and neurofibromatosis 2-related schwannoma and meningioma studied with PET. Eur J Nucl Med Mol Imaging 34: 87-94, 2007.

15. Deantonio L, Beldi D, Gambaro G, Loi G, Brambilla M, Inglese $\mathrm{E}$ and Krengli M: FDG-PET/CT imaging for staging and radiotherapy treatment planning of head and neck carcinoma. Radiat Oncol 3: 29, 2008.

16. Baek CH, Chung MK, Son YI, Choi JY, Kim HJ, Yim YJ, Ko YH, Choi J, Cho JK and Jeongn HS: Tumor volume assessment by ${ }^{18} \mathrm{~F}-\mathrm{FDP}$ PET/CT in patients with oral cavity cancer with dental artifacts on CT or MR Images. J Nucl Med 49: 1422-1428, 2008.

17. Busse PM, Harling OK, Palmer MR, Kiger WS III, Kaplan J, Kaplan I, Chuang CF, Goorley JT, Riley KJ, Newton TH, Santa Cruz GA, Lu XQ and Zamenhof RG: A critical examination of the results from the Harvard-MIT NCT program phase 1 clinical trial of neutron capture therapy for intracranial disease. J Neurooncol 62: 111-121, 2003.

18. Coderre JA, Morris GM, Kalef-Ezra J, Micca PL, Ma R, Youngs $\mathrm{K}$ and Cordon CR: The effects of boron neutron capture irradiation on oral mucosa: evaluation using a rat tongue model. Radiat Res 152: 113-118, 1999.

19. Aihara T, Hiratsuka J, Morita N, Uno M, Sakurai Y, Maruhashi A, Ono K and Harada T: First clinical case of boron neutron capture therapy for head and neck malignancies using ${ }^{18}$ F-BPA-PET. Head Neck 28: 850-855, 2006.

20. Miyashita M, Miyatake S, Imahori Y, Yokoyama K, Kawabata S, Kajimoto Y, Shibata MA, Otsuki Y, Kirihata M, Ono K and Kuroiwa T: Evaluation of fluoride-labeled boronophenylalaninePET imaging for the study of radiation effects in patients with glioblastomas. J Neurooncol 89: 239-246, 2008. 